\title{
LV-BEST-OP-3
}

\section{Characteristics of TP53 and CTNNB1 mutation according to the serum tumor markers in hepatocellular carcinoma}

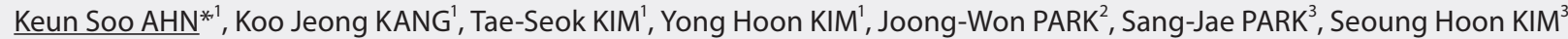 \\ 'Department of Surgery, Keimyung University Dongsan Hospital, Daegu, Korea \\ ${ }^{2}$ Department of Internal Medicine, National Cancer Center, Goyang, Korea \\ ${ }^{3}$ Department of Surgery, National Cancer Center, Goyang, Korea
}

Introduction: Serum alpha-fetoprotein (AFP), Lens culinaris agglutinin-reactive AFP (AFP-L3), and des-gamma-carboxyprothrombin (DCP) are useful biomarkers of hepatocellular carcinoma (HCC). However, associations among molecular characteristics and serum biomarkers are unclear.

Methods: We analyzed RNA expression and DNA variant data from The Cancer Genome Atlas Liver Hepatocellular Carcinoma (TCGA-LIHC) to examine their associations with serum biomarker levels and clinical data. From 371 TCGA-LIHC patients, we selected 91 seen at 3 institutions in Korea and the United States and measured AFP, AFP-L3, and DCP from preoperatively obtained serum. We conducted an integrative clinical and molecular analysis, focusing on biomarkers, and validated the findings with the remaining 280 patients in the TCGA-LIHC cohort.

Results: Patients were categorized into 4 subgroups: elevated AFP or AFP-L3 alone ( $\uparrow$ AFP\&L3), elevated DCP alone ( $\uparrow$ DCP), elevation of all 3 biomarkers ( $\uparrow$ All), and reference range values for all biomarkers (RR). CTNNB1 variants were frequently observed in $\uparrow$ DCP patients $(\mathrm{n}=7$ [53.8\%]) and RR patients $(\mathrm{n}=10$ [38.5\%]), but $\uparrow \mathrm{DCP}$ patients with a CTNNB1 variant had worse survival than RR patients. TP53 sequence variants were associated with $\uparrow$ AFP $(n=8$ [30.8\%]) and $\uparrow$ DCP $(n=4[30.8 \%])$. The Wnt signaling pathway was activated in the $\uparrow A F P \& L 3$, whereas liver-related Wnt signaling was activated in the RR. TGF and VEGF signaling are activated in high AFP\&L3, while dysregulated bile acid and fatty acid metabolism were dominant in $\uparrow \mathrm{DCP}$. We validated these finding using the remainder of the TCGA-LIHC cohort and showed similar results to the test cohort.

Conclusions: Serum AFP, AFP-L3, and DCP levels can help predict variants in the genetic profile. 